\title{
Los videojuegos como materiales curriculares: una aproximación a su uso en Educación Física Video games as curriculum materials: an approach to their use in Physical Education *Fernando Gómez-Gonzalvo, **Pere Molina, ** José Devís-Devís \\ *Universidad San Jorge (España), **Universitad de Valencia (España)
}

\begin{abstract}
Resumen. Los materiales curriculares son herramientas del profesorado para el desarrollo de sus clases que actúan como mediadores en el proceso de enseñanza-aprendizaje. Desde inicios del siglo XXI se ha desarrollado un cuerpo teórico sobre el uso de videojuegos como material curricular, pero su uso presenta beneficios e inconvenientes por lo que se han generado importantes lagunas en cómo deben usarse como material educativo. El presente trabajo pretende analizar el potencial de los videojuegos como material curricular para la asignatura de Educación Física. Para ello, se profundiza en los tipos de usos que pueden derivarse de los videojuegos en el desarrollo del currículum. Se ofrecen ejemplos de uso de videojuegos como material curricular tanto desde una racionalidad técnica como desde una racionalidad práctica. Por último, se bosquejan usos posibles de los videojuegos y se señalan las perspectivas de futuro del uso de los videojuegos para la asignatura de Educación Física.

Palabras clave: Material curricular, videojuegos, TIC, racionalidad práctica, profesionalización docente.
\end{abstract}

\begin{abstract}
Curricular materials are teachers' tools used for their teaching development which mediate the teaching-learning process. At the beginning of the 21st century a theoretical body of knowledge has been developed on the use of video games as curricular material, but its use has benefits and disadvantages, which produce important gaps on how they should be used. This paper analyzes the potentiality of video games as curriculum materials for the Physical Education subject. Therefore, we analyze in depth on the types of use video games offer in curriculum development. Several examples of video games use are presented from a technical and practical rationality. Finally, possible uses are drawn and future perspectives on video games usage for Physical Education are indicated.
\end{abstract}

Key words: Curriculum material, Video games, ICT, Practical rationality, teacher professionalization.

\section{Introducción}

Los materiales curriculares son herramientas que sirven al profesorado en el proceso de enseñanza como mediadores entre el currículum y la práctica educativa y que representan un vínculo con las teorías curriculares (Molina, Devís y Peiró, 2008). Esta concepción amplia de material curricular engloba desde los materiales escritos (libro de texto, programaciones didácticas, unidades didácticas, fichas de sesiones, etc.) hasta los recursos materiales (instalaciones, equipamiento y materiales deportivo), así como los medios audiovisuales e informáticos (proyectores, ordenadores, tablets, etc.).

Aunque encontramos materiales que originariamente han sido creados como curriculares (un claro ejemplo son los libros de texto) otros no han sido concebidos desde un principio para tal propósito (como puede ser un periódico o un videojuego). Sin embargo, estos últimos pueden convertirse en materiales curriculares si se utilizan con una finalidad pedagógica dentro del contexto escolar. Así pues, el potencial curricular de los materiales va más allá de ser un aspecto inherente a los mismos, y depende del uso que se haga de ellos. El uso que se haga de un material es el aspecto clave para desarrollar su potencialidad curricular (Molina, Devís y Peiró, 2008; Molina, Martínez-Baena, y Gómez Gonzalvo, 2017).

Se ha escrito mucho sobre materiales curriculares, pero la utilización del videojuego, por su reciente uso, no ha sido abordada extensamente y menos en Educación Física, por lo que existen muchas lagunas e incertidumbres sobre su uso en el contexto de esta asignatura. Lo que sabemos es que los videojuegos forman parte de la vida de los adolescentes desde la década de 1980. Desde entonces las videoconsolas comienzan a introducirse en todos los hogares y se crean los primeros salones recreativos, incrementando así la difusión de los videojuegos (Belli y López, 2008).

Durante la primera década del siglo XXI se empieza a desarrollar un cuerpo teórico de cierta importancia en el que se reivindica el uso de videojuegos como material educativo (Etxeberría, 2012; Griffiths, 2002; Gros, 2007, 2008 y 2009). El informe Horizont de 2012 señalaba que tanto los videojuegos como sus dinámicas se introducirían en los entornos educativos a través de la gamificación de los contenidos (Durall, Gros,

Fecha recepción: 15-01-18. Fecha de aceptación: 22-02-18 Fernando Gómez-Gonzalvo fgomez@usj.es
Maina, Johnson, y Adams, 2012). De hecho, su uso como material curricular está aumentando en los últimos años por parte del profesorado de todos los niveles educativos debido a las posibilidades educativas que ofrecen.

El profesorado de todas las asignaturas está integrando este tipo de material en sus clases, incluido el de Educación Física, a pesar de la percepción negativa que existe por parte de este colectivo hacia las posibilidades educativas del videojuego (Jenny, Schary, Noble y Hamil, 2017). Por ello, el objetivo de este artículo es analizar el potencial de los videojuegos como material curricular en la asignatura de Educación Física. Para ello, se profundiza en los tipos de usos que se pueden derivar de los videojuegos mostrando sus posibilidades curriculares.

\section{Formas de uso de los materiales curriculares: los} videojuegos

La literatura sobre materiales curriculares identifica dos formas básicas en su uso: a) el uso de materiales como elementos neutros; y b) el uso de materiales como elementos de experimentación (Area, 1999, 2004; Devís et al., 2001; Devís y Peiró, 2004; Guarro, 2005; Martínez Bonafé, 1991a, 1991b; Molina, Martínez-Baena y Gómez-Gonzalvo, 2017; Peiró y Devís, 1994).

Los materiales como elementos neutros transmiten información y construyen conocimiento, tal y como han sido configurados por las empresas que los elaboran. El profesorado queda relegado a un usuario de un contenido curricular concreto y su papel consiste en seleccionar aquellos materiales que considera idóneos para desarrollar su práctica educativa. El material integra las tareas y contenidos de la manera que deben desarrollarse en clase. De este modo, se homogeneiza y unifica el currículum educativo dejando de lado la adaptación al contexto concreto de cada aula. Una de las características de este tipo de materiales es que pueden utilizarse en cualquier centro, con cualquier tipo de alumnado y por cualquier profesor o profesora. Es por ello que se les conoce como materiales ‘a prueba de profesores' porque limitan la práctica docente de tal manera que el profesorado puede llegar a ser sustituido por el material que señala los ejercicios y tareas a desarrollar (Apple, 1989). Desde esta concepción, el profesor delega el control de la enseñanza a los materiales que utiliza y el propio material deja de ser un medio para convertirse en la finalidad educativa.

Esta forma de uso de los materiales curriculares se sustenta en una racionalidad técnica, donde el proceso de enseñanza-aprendizaje resulta 
de la manifestación de la eficacia a través de materiales y técnicas. Busca la consecución del éxito educativo en términos de rendimiento, es decir, aprender más en el menor tiempo posible, y que además pueda observarse y medirse. El profesorado queda relegado a un mero aplicador de materiales y contenidos pensados y elaborados por otros que resultan ajenos a su realidad educativa.

Por su parte, los materiales curriculares entendidos como elementos de experimentación requieren de la intervención del profesorado. El profesor no puede ser un elemento ajeno al uso del material. Desde esta concepción, los materiales no se usan con el alumnado, sin más, sino que requieren de una adaptación previa por parte del profesorado que es quien decide qué material se utiliza, cuándo se hace y en qué sentido se trabaja con él. En otras palabras, es el profesorado quien decide la metodología y las condiciones en las que se usan los materiales dentro de las necesidades pedagógicas y el contexto particular de la enseñanza. Los materiales no son más que un medio y un punto de partida que tienen un carácter provisional.

Este tipo de uso de los materiales curriculares se sustenta en una racionalidad práctica que concibe la enseñanza-aprendizaje como un proceso contextualizado en una realidad socioeducativa concreta. En esta situación el profesorado experimenta, reflexiona y redirige la práctica hacia las diversas necesidades del alumnado. Desde esta racionalidad, el profesorado se postula como un profesional que, además de la competencia técnica, tiene una conciencia moral, una intencionalidad política y una implicación emotiva que forma parte de su labor profesional (Hargreaves, 1995). El proceso educativo se entiende como una actividad incierta, singular y en conflicto con los diferentes agentes que participan en la enseñanza. Esto requiere del profesorado un conocimiento implícito de la actividad docente, una reflexión in situ sobre lo que se realiza y un feedback posterior para la reorganización de las actividades docentes (Schön, 1992).

\subsection{Los videojuegos como materiales 'a prueba de profesores'}

Los videojuegos se han mostrado como herramientas eficaces a la hora de aprender y se han encontrado beneficios en su utilización en contextos diversos. Por ejemplo, se han mostrado efectivos para la regulación de conductas en personas con trastornos de la personalidad y se han encontrado mejoras en las habilidades de comunicación, en el control de conductas impulsivas, en la reducción de ansiedad, el autocontrol y en la reducción de conductas autodestructivas (Etxeberría, 1998). Por otro lado, se han mostrado eficaces para el desarrollo de habilidades cognitivas, psicomotoras, sociales, emocionales y lingüísticas (Griffiths, 2002; Rosas et al., 2003; Gee, 2004). Incluso se han encontrado efectos positivos en la mejora de la atención, en la búsqueda, organización y asimilación de información, en la creatividad y en el proceso de toma de decisiones (Gros, 2007).

En la bibliografía actual existe un nutrido volumen de propuestas en las que se integran los videojuegos en las aulas y, cabe destacar que, la mayoría de ellas se han realizado con la utilización de videojuegos comerciales (Lacasa, 2011; Del Moral y Fernández, 2012; Revuelta y Guerra, 2012). El uso de videojuegos se ha propuesto para diseñar las intervenciones educativas en la búsqueda de la máxima eficacia didáctica y de reflexionar sobre el propio diseño del videojuego (Marqués, 2001; Del Moral, Villalustre, Yuste y Esnaloa, 2010). Desde este punto de vista, resulta importante su selección y ésta se realiza atendiendo a sus requerimientos técnicos y al alumnado al que van dirigidos, según la clasificación PEGI (Pan European Game Information) de sus contenidos. Además, se requiere que pueda evaluarse el avance del alumnado a través de los retos del propio videojuego (Yuste, 2012).

Según esta perspectiva, el uso pedagógico de los videojuegos se centra en la propia herramienta y en las habilidades que pueden desarrollarse con su uso, tal y como sostienen Del Moral y Fernández (2012). Según estas autoras, la elección de los videojuegos debe fundamentarse en los objetivos que el videojuego nos ofrece. Es decir, el profesorado debe prestar atención al tipo de habilidades o tareas que nos propone la herramienta con el fin de seleccionar la que mejor y más eficazmente desarrolle este tipo de habilidades. También debe prestarse atención al tipo de contenidos que ofrece el videojuego de forma que sean estos los que guíen nuestra práctica educativa. Así, la labor del profesorado ha de consistir en adaptar la práctica educativa a los contenidos de los videojuegos, profundizando en ellos a través de trabajos complementarios. Se trata de desarrollar las competencias del alumnado con la ayuda de los videojuegos mientras que el profesorado se ocupa de la gestión y organización de la clase.

La utilización de los videojuegos como material curricular se sustenta, principalmente, sobre la idea de que son actividades placenteras con un gran componente lúdico que ‘engancha' al alumnado (Del Moral, 2004). Sin embargo, Dorado y Gewec (2017) señalan que esta forma de utilización de los videojuegos cuenta con una importante limitación. Estas autoras indican que la forma predominante de enseñanza que subyace en los videojuegos es de tipo conductista o neo-conductista. Es decir, se focaliza la atención en los contenidos del videojuego para memorizar los datos reduciendo las posibilidades de participación e interacción en el juego, de forma similar a cómo se utilizan los libros de texto. Además, los métodos neo-conductistas no sólo se utilizan en los contenidos que desarrollan los videojuegos, sino que las bases de programación del juego también siguen estas dinámicas (Del Olmo, 2013).

Deacuerdo con estas autoras, podemos entender que los videojuegos son una evolución de la máquina de enseñanza de Skinner (1958), mucho más elaborada y dinámica, pero con unas bases de aprendizaje comunes. Esta máquina era una caja electrónica a través de la cual se le presentaba preguntas de verdadero-falso o de respuesta múltiple al alumnado y debía de responderlas para pasar a la siguiente. Esta máquina utilizaba el refuerzo positivo como mecanismo de recompensa. Cuando el alumnado acertaba la respuesta se encendía una bombilla verde y se reproducía un sonido agradable. En cambio, cuando la respuesta era incorrecta se encendía una bombilla roja y se escuchaba un sonido desagradable. La máquina se podía 'ajustar al aprendizaje’ con bobinas intercambiables de preguntas adaptándolas al nivel educativo. El avance del alumnado se entendía de forma que, cuantas más repuestas correctas era capaz de contestar, más aprendizaje se producía porque había acumulado conocimiento. La máquina daba un feedback inmediato sobre el resultado y favorecía que el alumnado aprendiera de manera eficiente a hacer bien la tarea. Además, le mantenía motivado ante la tarea porque el trabajo era placentero por el aumento del nivel de interés y de entusiasmo.

Tanto en la máquina de enseñanza como en los videojuegos, al alumnado se le presenta un reto, en forma de objetivo a superar, y a través de las interacciones con la máquina se le guía hacia la respuesta correcta. En el caso de la máquina de enseñanza se utilizaban luces y sonidos para reforzar positivamente al estudiante cuando conseguía la respuesta correcta. En el caso de los videojuegos, a medida que se va desarrollando la historia se recogen objetos especiales o ítems que forman parte del inventario que va utilizándose para completar el propio videojuego. De esta manera se crea una retroalimentación positiva en la propia tarea que hace aumentar la motivación y las ganas de continuar en ella, tal y como explican Salas y Lewis (2013). Además, se ha encontrado otro tipo de refuerzo positivo que se obtiene por parte de la comunidad de jugadores y que se da a la hora de desbloquear pantallas o 'armas', conseguir logros predefinidos por la máquina y obtener puntuaciones altas con las que compararse en las clasificaciones (Cortizo et al., 2011).

Desde esta perspectiva, la superación de niveles de juego, el alcance de retos y el avance en la historia es un reflejo del desarrollo de aprendizajes. Pero, ¿hasta qué punto el nivel del jugador en el juego puede traducirse en aprendizajes? Es como cuando se utiliza mecánicamente un libro de texto, ¿ hasta qué punto el avance en el libro puede traducirse en aprendizajes? Consideramos que existen grandes limitaciones a la hora de conectar el avance del juego con los aprendizajes, con la evaluación y con la calificación del alumnado. En el caso de los actuales videojuegos comerciales, resulta todavía mucho más complicado puesto que avanzar más o menos rápido en la historia no sólo depende del posible aprendizaje que realice el alumnado. La gran comercialización de los videojuegos y su explotación como opción de entretenimiento ha 
generado un nuevo hito comercial desconocido hasta el momento. Los videojuegos actuales requieren de compras on-line y actualizaciones periódicas para poder jugar y poder continuar en la historia. Esta comercialización incluye las 'armas' especiales que hacen más fácil progresar, el desbloqueo de habilidades o magias de los personajes y las compras de vidas extra que van facilitando la tarea. En otras palabras, puede avanzarse en el juego gastando dinero real sin necesidad de esforzarse porque los pagos permiten conseguir las soluciones a los problemas que nos presenta el juego.

Un análisis detallado entre los videojuegos y el libro de texto nos presenta varias similitudes importantes. En primer lugar, los videojuegos son materiales elaborados por expertos ajenos a la realidad educativa que organizan y planifican los videojuegos basándose en elementos comerciales (Cebrián, 2013). En segundo lugar, al profesorado le resulta imposible modificar los contenidos de los videojuegos para adaptarlos a su realidad docente. Los conocimientos de programación necesarios para su modificación y la falta de recursos requieren un esfuerzo extra por parte del profesorado que, por otra parte, ya se encuentra en una situación de intensificación docente. Todo ello sin olvidar que los conocimientos informáticos requeridos exceden la formación del profesorado, convirtiendo el uso de videojuegos en una tarea imposible de realizar desde esta perspectiva (Fernández-Pampillón, Domínguez y Armas, 2012). Una forma de superar estas limitaciones ha sido la creación de vías de colaboración entre los creadores de videojuegos y el profesorado (Dorado y Gewec, 2017), pero estas vías alternativas recuerdan a las ya utilizadas por las editoriales de libros de texto para superar parte de sus limitaciones.

La imposibilidad de modificación de los videojuegos se traduce en una homogenización de los contenidos curriculares porque el profesorado no puede adaptar ni modificar aquello que va a realizar en clase. Las adaptaciones curriculares no son factibles puesto que los retos predefinidos por el código informático son iguales para todo el alumnado. A pesar de que la programación de los videojuegos puede llegar a adaptar automáticamente los retos al nivel de los jugadores no podemos hablar de una adaptación curricular como tal porque no se adapta el material en sí, sino aquello que el material realiza. Es decir, ocurre algo muy parecido a lo que ocurría con la máquina de la enseñanza de Skinner en la que se podían cambiar las bobinas de preguntas adaptándolas al nivel del alumnado, pero la forma de preguntar y el feedback que devolvía la máquina eran idénticos, independientemente del nivel o las necesidades educativas que podía presentar el alumnado. Por ejemplo, si el videojuego presenta un puzzle para resolverlo éste puede tener 5,10 , 20 o 30 piezas (adaptado al nivel del alumnado), pero si el alumnado presenta dificultades educativas que le dificulta realizar el puzzle no se puede cambiar la tarea por clasificar figuras geométricas puesto que el profesorado no tiene poder para transformar la herramienta.

\subsection{Los videojuegos como elementos de experimentación del pro-} fesorado

Los videojuegos se definen como campos no-lineales y multicursales porque no tienen una secuencia establecida y pueden (re)dirigirse hacia múltiples finales o soluciones (Juul, 2005). Seentiende que los videojuegos presentan una estructura de simulación que se construye a través de la utilización de diferentes recursos (literarios, ergódicos, lúdicos, etc.) y que configuran un espacio donde se desarrolla una historia (Frasca, 2003). En este sentido, no es una estructura cerrada donde se tiene cierta previsión de qué va a pasar y cómo va a pasar. Por ello, los videojuegos pueden enfocarse como materiales curriculares para la experimentación.

Gros (2009) afirma que el uso de videojuegos para favorecer el aprendizaje no es sólo un instrumento más para transmitir conocimientos sino un pretexto para transformar el modelo educativo hacia la práctica. En este sentido, el profesorado debe de contextualizar el uso de videojuegos dentro de su programación educativa, con unos objetivos pedagógicos concretos y aprovechando su potencial experimental para complementar las necesidades pedagógicas del profesorado y del alumnado (Etxeberría 2012; Gee, 2004; Gros, 2008).
Desde este punto de vista, puede introducirse al alumnado a la investigación de soluciones en vez de a la reproducción de tareas. Los jugadores deben aplicar conocimientos formales, informales y propios del videojuego para resolver los problemas presentes en ellos según la experiencia práctica acumulada en el juego(Dabbagh y Kitsantas, 2012). Dado que la resolución de los problemas y las consecuencias derivadas se realizan en un plano virtual, éstas no tienen serias implicaciones sobre la realidad, pueden proponerse diferentes alternativas, por muy abstractas e imposibles que parezcan puesto que nadie resultará herido. Además, su uso debe basarse en la posibilidad de autoevaluar los resultados y corregir los errores de forma que se cree un ciclo de aprendizaje mediante la búsqueda de soluciones y evaluación de resultados (Gros, 2007)

No debemos olvidar que debe analizarse críticamente lo desarrollado en el videojuego y esto ha de servir para construir conocimientos válidos y superar las limitaciones y barreras que presentan. La experimentación puede realizarse en el plano virtual, pero debe relacionarse con la realidad, con hechos históricos reales o con hechos científicos para construir un conocimiento válido (Sánchez Agustí, 2005).

El papel del profesorado en el uso de videojuegos como material de experimentación requiere supeditar los videojuegos a la figura del profesorado y que éstos sean un complemento a la labor docente (Gros, 2008). En este sentido, son un material curricular más que debe estar cargado de sentido e intencionalidad para su introducción en el proceso de enseñanza-aprendizaje. En ningún caso han de sustituir al profesorado en su labor, sino que éste debe utilizar los videojuegos para experimentar alguna parte de los contenidos. A pesar de las limitaciones del profesorado para modificar y reestructurar este tipo de materiales, debido a los elevados conocimientos informáticos que requiere, su labor de seleccionador y de evaluador del material es fundamental.

Por último, la introducción de los videojuegos dentro del contexto educativo está evolucionando de tal manera que no se limita al uso comercial del videojuego sino al aprovechamiento del propio proceso de los videojuegos mediante lo que se conoce como gamificación. La gamificación serefiere a la aplicación del juego dentro de las dinámicas de clase y en actividades que no tienen una intención originalmente lúdica. En otras palabras, es una metodología que integra las dinámicas de juego a través de cualquier soporte, digital o no, con el objetivo de aumentar la participación, motivar al alumnado, desarrollar competencias basadas en el aprendizaje analítico y de resolución de problemas para trabajar los contenidos educativos como si fuera un juego (Durall et al., 2012). Las experiencias de gamificación han sido construidas utilizando las dinámicas y características de los videojuegos, pero no necesariamente utilizando un producto comercial.

\section{El uso de los videojuegos en Educación Física}

El campo de los videojuegos ha experimentado grandes avances en los últimos años y su uso como material curricular está determinado, en parte, por el avance del desarrollo tecnológico. Recordemos que los videojuegos no sólo han de entenderse desde un punto de vista convencional, en el que la interacción humano-máquina se realiza con un mando, sino también a través del cuerpo con el desarrollo de videoconsolas y videojuegos que capturan el movimiento humano y lo integran en el videojuego. Las videoconsolas Wii o Kinect han roto la visión tradicional de los videojuegos y se han liberado del mando para manejar al personaje del juego. Digamos que la interacción humano-máquina ha substituido el mando por el movimiento corporal para crear experiencias de juego más realistas.

Los videojuegos se han utilizado en diferentes estudios para desarrollar los contenidos curriculares de esta asignatura. En el trabajo de revisión de Jenny, et al. (2017) se señala que los videojuegos en Educación Física se han utilizado como herramienta para aumentar la motivación y la participación en clase, pero existen grandes posibilidades para el desarrollo de habilidades motrices a través de los videojuegos que el profesorado desconoce. 


\subsection{Ejemplos de uso de videojuegos 'a prueba de profesores'}

Como hemos señalado anteriormente, existen experiencias en Educación Física con el uso de videojuegos que han estado enfocadas como materiales de uso neutro. En su experiencia, Del Castillo et al. (2012) utilizaban videojuegos deportivos (FIFAy NBA) en clase y mostraron cómo el alumnado aprendía las normas básicas de funcionamiento del propio videojuego y del reglamento del deporte que simulaban. De este modo, se abordaban contenidos de la asignatura de Educación Física en el videojuego y el objetivo didáctico consistía en el aprendizaje de las normas que rigen el juego y que se desarrollan en él. En este estudio se evidencia que el aprendizaje se realizaba a través del videojuego y de su experiencia de juego, principalmente a través del feedback negativo o neutro que devuelve el juego en forma de faltas o sanciones y buenas jugadas. También pueden utilizarse estos videojuegos de deporte para el aprendizaje de las estrategias de juego. Por ejemplo, puede seleccionarse un partido con diferentes formaciones de juego en fútbol (4-4-2; 3-5-15; etc.) o los tipos de defensa en baloncesto (en zona, individual, presionante, box-one, etc.) con el objetivo didáctico de conocer las diferentes formaciones que existen y saber cómo se utilizan en el juego. En este trabajo, el profesorado servía de guía en los videojuegos y ayudaba al alumnado en la gestión de estrategias para jugar.

Otro ejemplo de uso de videojuegos son los de bailes como las sagas Just Dance o Dance, dance revolution que podrían encajar en los contenidos de danza y expresión corporal. En este sentido, George, Rohr, y Byrne (2016) encontraron que se aumentaban las habilidades motrices individuales a través de videojuegos de bailes. Una manera de introducir los videojuegos en la asignatura puede ser utilizándolos como medio para perder la vergüenza en este tipo de actividades y para el trabajo coreografiado. El propio videojuego marca el ritmo de ejecución y los pasos que el alumnado ha de aprender y seguir, con lo que el profesorado únicamente debe seleccionar el videojuego, gestionar los recursos y la dinámica de clase.

También existen ejemplos que utilizan algunos videojuegos para medir el consumo calórico entre videojuegos activos y no activos o entre los videojuegos y el gasto energético de la actividad real (Beltrán-Carrillo, Valencia-Peris y Molina, 2011). En su aplicación a la Educación Física, existen multitud de programas que se han desarrollado con relojes (wachfitness) o steps (Wii fit) que utilizan acelerómetros para medir los pasos realizados, las calorías consumidas, los pasos restantes para superar el siguiente nivel de actividad física o muestran baterías de ejercicios para la mejora de la salud. Se trata de programas de actividad física orientada a la salud en las que el usuario sigue las instrucciones programadas por expertos. Este sistema utiliza todos los refuerzos positivos de los videojuegos como puntuaciones, clasificaciones, control de objetivos, premios y ejercicios para realizar actividad física con el fin de mejorar la salud.

\subsection{Ejemplos de uso de videojuegos como elementos de experi-} mentación

En el uso de videojuegos como elementos de experimentación resulta fundamental la aplicación, la contextualización y el análisis que realice el profesorado. Esto implica la existencia de diversos niveles de experimentación en el uso de videojuegos en Educación Física. Los ejemplos que proponemos a continuación son una muestra de los tres niveles de experimentación que hemos considerado ejemplificar. El primer nivel de experimentación, el nivel inferior, puede darse cuando el profesorado selecciona el videojuego y trabaja sobre él, con grandes niveles de auto-aprendizaje por parte del alumnado, pero con un rol importante del profesorado en el feedback y la evaluación. El segundo nivel o nivel intermedio surge cuando el profesorado utiliza las estrategias y características del videojuego, a través de la gamificación, para el desarrollo de parte o del conjunto de contenidos curriculares de la asignatura. El nivel superior de experimentación surge cuando el profesorado y el alumnado conjuntamente intervienen en el proceso de creación de videojuegos o gamificaciones contextualizadas a su realidad.

Como primer ejemplo del nivel inferior tenemos la introducción en nuestras clases de un videojuego como el FIFA(siguiendo el ejemplo del punto anterior) que permite jugar partidos de fútbol con diferentes formaciones de juego. Este uso requeriría analizar las ventajas y desventajas de cada formación y cómo podemos utilizarlas en la práctica real. Incluso, profundizando aún más, podrían plantearse actividades en las que el alumnado experimente el videojuego para analizar posteriormente las decisiones tácticas que se han adoptado, comprobar si han sido adecuadas y proponer soluciones. Una experiencia en este sentido es el entrenamiento a través de ejercicios que faciliten la puesta en marcha de habilidades perceptivas y toma de decisiones a través de videojuegos donde no se acumula el cansancio de las acciones motrices (Shafer, Carbonara y Popova, 2011)

Un segundo ejemplo denivel intermedio de experimentación serían propuestas similares a las presentadas por Pérez-López, Rivera y Trigueros (2017) y Pérez-López y Rivera (2017) en las que gamifican los contenidos de la asignatura de Didáctica de la Educación Física del grado deCiencias delaActividad Física y el Deporte. En esta gamificación se utiliza la dinámica de un juego de rol, inspirándose en la serie televisiva de 'juego de tronos'. Aquí el alumnado, por grupos, debe defender 'un reino’ de la Educación Física que son los cuatro principales bloques de contenido de la asignatura (Físicor, condición física; Deporticia, deportes y juegos tradicionales; Expresanto, expresión corporal y danza y; Naturalia, actividades en el medio natural). El juego se realiza a través de desafíos y retos en los que los integrantes de cada reino deben desarrollar las habilidades de enseñanza de la Educación Física. Cada reino tiene habilidades concretas y se les premia con puntos cada vez que resuelven un desafío, lo cual determina diferentes bonificaciones y contraprestaciones dependiendo del resultado. El objetivo final es conquistar el nivel superior de puntuación y con ello 'el trono de los cuatro reinos'. El profesor participa creando los desafíos y retos y, además, se asigna la figura de 'oráculo de los dioses' para resolver cualquier disputa que pueda surgir.

Por último, el tercer nivel de experimentación sería aquel en el queel profesorado diseñara sus propios videojuegos o gamificaciones con un objetivo educativo centrado en su contexto, como señalan Dorado y Gewec (2017). Se trataría de abordar un problema de su práctica educativa, en el cual tuviera el control de los contenidos que desarrolla, de la perspectiva desde la que abordarlos, del análisis posterior y de la comprobación del material como válido para ese contexto. En otras palabras, un profesorado autónomo con un alto dominio de herramientas tecnológicas, metodología e innovación que sea capaz de aplicarlas en sus clases. Asi, por ejemplo, el profesor o la profesora puede elaborar un videojuego sobre la historia de los juegos olímpicos con la ayuda de las plataformas en línea como 'e-adventure' que no requiere grandes conocimientos informáticos. De esta manera, el desarrollo del juego puede utilizar acertijos, adivinanzas, puzzles u objetos a encontrar y coleccionar para aprender sobre los juegos olímpicos. Un paso más allá en este nivel más alto sería que el alumnado interviniera en crear parte del videojuego y darle sentido dentro de su contexto educativo y centrado en sus intereses. Así el alumnado sería una figura autónoma y activa, implicada en su propio proceso de aprendizaje a través de la creación de materiales curriculares para él mismo y para sus compañeros, es decir, que el alumnado adopte roles de responsabilidad ante la asignatura. Un ejemplo desde la participación del alumnado sería la exposición de trabajos de clase a través del videojuego, en lugar de recurrir al típico trabajo escrito, en el que el hilo conductor del juego sean los contenidos del tema que el alumnado ha de desarrollar y aprender.

\section{Comentarios finales}

El uso de videojuegos como material curricular, no sólo en Educación Física sino en todas las asignaturas, se ha realizado principalmente desde una racionalidad técnica. Es decir, con un uso más o menos mecánico de los videojuegos que intensifica el trabajo del profesorado (Molina, Martínez-Baena, Gómez-Gonzalvo, 2017). La mayoría de experiencias de videojuegos en educación han resultado formas de uso como materiales 'a prueba de profesores'. El profesorado ha quedado supeditado a la aplicación de videojuegos desarrollados por la industria 
que ofrecen una serie de contenidos y retos a superar de forma lúdica. Esto ha facilitado la preparación y programación de contenidos debido a la falta de tiempo ocasionada por el aumento de las labores administrativas. Este enfoque ha aumentado el proceso de tecnificación y burocratización de la labor docente, sustituyendo parte de las tareas propias del profesorado por tareas puramente administrativas y de gestión de la clase (Gimeno, 1988; Kirk, 1990; Blanco, 1994).

Para que los videojuegos puedan ser utilizados como material curricular requiere, como otro tipo de materiales, un enfoque pedagógico con unos objetivos y utilidad educativa clara. Tradicionalmente, los materiales (sobre todo libros de texto) se han utilizado como herramientas para suministrar información (Molina, 2015), pero los videojuegos no cumplen ese objetivo. Son capaces de trasladar al alumnado a un contexto histórico, deportivo, socio-cultural o geológico simulado para trabajar en la compresión de qué hacer, cómo hacer y por qué hacer una acción con el fin de solucionar un problema. Las críticas y limitaciones de los videojuegos como herramienta educativa se justifican cuando el videojuego pretende sustituir al profesorado y cuando se requiere profundidad en el tema, pero no cuando la pretensión es presentar y enlazar lo que se experimenta en el videojuego con la realidad para obtener un conocimiento válido y significativo sobre temas generales, como puede ser en la Educación Primaria y Secundaria (Sánchez Agustí, 2005).

El carácter práctico de la asignatura de Educación Física no debe confundirse con el uso de los materiales curriculares desde una perspectiva práctica o experimental. En la asignatura se manifiestan unas tradiciones pedagógicas que están sujetas a la cultura física que la domina (Molina et al., 2008) y su carácter práctico puede llevar a confusión con un uso de materiales como elementos de experimentación (Molina et al. 2017). En este sentido, en el uso de videojuegos ha predominado un tipo de actividad técnica que se ha centrado en la eficiencia educativa, pero en pocos casos, se ha planteado su uso como material para la experimentación. Su uso se ha fundamentado en la mejora de contenidos relacionados con los deportes, en la mejora de las habilidades motoras y perceptivas como simples tareas y ejercicios físicos (Jenny et al., 2017).

No obstante, debe enfocarse el uso de videojuegos como un medio para la experimentación, utilizando los recursos especiales que ofrecen para la asignatura de Educación Física. Es decir, facilitar experiencias que requieran un análisis sobre lo que ha sucedido en el juego para profundizar en los contenidos curriculares, mediante la estrategia metodológica de la gamificación de los contenidos o con la participación del alumnado y el profesorado en la creación de videojuegos como material didáctico. Estas formas de uso permiten que los materiales se adapten a necesidades educativas concretas del contexto de enseñanzaaprendizaje de clases particulares. De esta manera, el uso de los videojuegos o la gamificación entendidos como medio de experimentación puede tener un largo recorrido en la Educación Física escolar.

Por último, cabe señalar, como reflexión para el futuro, la irrupción de un grupo especial de videojuegos con raíz deportiva. Nos referimos al desarrollo de los e-sports, que no únicamente se refieren a los juegos deportivos sino a una práctica deportiva o deportivizada dentro de un videojuego. Actualmente existen grandes competiciones promovidas por la industria del videojuego en las que se compite por un título mundial o por premios en metálico de forma similar a los deportes no electrónicos. Creemos, de hecho, que ha existido un proceso de deportivización dentro de la industria del videojuego que ha posibilitado este tipo de eventos de forma similar al proceso de deportivización de las actividades física en el siglo XIX. Consideramos que los e-sports deben ser objeto de estudio por parte de nuestro campo por las posibilidades que pudieran ofrecer a la hora de desarrollar algunas habilidades psicomotrices, por su componente socializador y por sus posibilidades de generar aprendizajes a través de la experiencia. También debemos pensar en el potencial inclusivo de los e-sports que no poseen los deportes tradicionales porque jugar en un espacio no real iguala a todos los participantes. De hecho, es posible competir en igualdad de condiciones traspasando la barrera institucional deportiva que ejerce su influencia como elemento segregador. De esta forma puede jugar juntos personas independientemente de su grado de movilidad, edad, sexo o incluso personas de cualquier nacionalidad sin cupos de extranjeros.

\section{Referencias}

Apple, M. W. (1989). Maestros y textos. Una economía política de las relaciones de clase y de sexo en educación. Barcelona: Paidós/ MEC.

Area, M. (1999). Los materiales curriculares en los procesos de diseminación y desarrollo del currículum. En J.M. Escudero (Ed.), Diseño, desarrollo e innovación del currículum (pp. 189-208). Madrid: Síntesis.

Area, M. (2004). Los medios y las tecnologías en la educación. Madrid: Pirámide.

Beltrán-Carrillo, V:; Valencia-Peris, A., \& Molina-Alventosa, J.P.(2011). Los videojuegos activos y la salud de los jóvenes: revisión de la investigación. Revista Internacional de Medicina y Ciencias de la Actividad Física y el Deporte, 10(41), 203-219.

Belli, S. y López, C. (2008). Breve historia de los videojuegos. Atenea Digital, 14, 159-179.

Blanco, N. (1994). Materiales curriculares: los libros de texto. En J.F. Angulo y N. Blanco (Coords.), Teoría y desarrollo del currículum (pp. 263-279). Archidona: Aljibe.

Cebrián, M. (2013). Juegos digitales para procesos educativos. En J.I. Aguaded y J. Cabero (coords.), Tecnologías y medios para la educación en la e-sociedad (pp. 185-208). Sevilla: Alianza editorial.

Cortizo et al. (2011). Gamificación y Docencia: Lo que la Universidad tiene que aprender de los Videojuegos. VIII Jornadas Internacionales de Innovación Universitaria. Villaviciosa de Odón. Recuperado de: http://hdl.handle.net/11268/1750

Dabbagh, N., \&Kitsantas, A. (2012).Personal Learning Enviroments, social media and self-regulatd learning: A natural formula for connecting formal and informal learning. Internet and higher education, 15,3-8. doi:10.1016/j.iheduc.2011.06.002

Del Castillo, H.; Herrero, D.; García, A.B.; Checa, M., \&Monjelat, N. (2012). Desarrollo de competencias a través de los videojuegos deportivos: alfabetización digital e identidad. RED. Revista de Educación a Distancia,33, Recuperado de: http://www.um.es/ead/red/ 33/delCastillo_et_al.pdf

Del Moral, E.; Villalustre, L.; Yuste, R., \&Esnaloa, G. (2010). Evaluación y diseño de videojuegos: generando objetos de aprendizaje en comunidades de práctica. RED. Revista de Educación a Distancia, 33, Recuperado de http://www.um.es/ead/red/33/esther_et_al.pdf

Del Moral, M.E. (2004). Pautas procedimentales para el diseño y análisis de videojuegos desde una perspectiva educativa. En E. Del Moral (coord.). Sociedad del Conocimiento, Ocio y Cultura: Un enfoque interdisciplinar (pp.407-426). Oviedo: Editorial KRK.

Del Moral, M.E., \& Fernández, L. (2012). Comunidades virtuales de videojugadores: Comportamiento emocional y social en poupéegirl. RED. Revista de educación a distancia, 33. Recuperado de: http:// www.um.es/ead/red/33

Del Olmo, D. (2013). Acercamiento al aprendizaje conductual bajo el mundo 1--1 de Super Mario Bros. Lifeplay, 1. Recuperado de: https://goo.gl/fHD1bq

Devís, J., \&Peiró, C. (2004). Los materiales curriculares en la educación física. En Fraile, A. (coord.), Didáctica de la educación física. Una perspectiva crítica y transversal (pp. 63-94). Madrid: Biblioteca Nueva.

Devís, J.; Peiró, C.; Molina, P.; Villamón, M.; Antolín, L., \& Roda, F. (2001). Los materiales curriculares impresos en educación física: clasificación, usos e investigación. Movimento, II(15), 116-136.

Dorado, S., \&Gewec, A. (2017). El profesorado español en la creación de materiales didácticos: los videojuegos educativos.Digital education rewiew, 31, 176-195.

Durall, E.; Gros, B.; Maina, M.; Johnson, L., \& Adams, S. (2012). Perspectivas tecnológicas: educación superior en Iberoamérica 2012-2017. Austin: The New Media Consortium. 
Etxeberría, F. (1998). Videojuegos y educación. Comunicar, 10, 171180.

Etxeberría, F. (2012). Videojuegos: riesgos y oportunidades en educación. Actas I congreso internacional videojuegos yeducación. Alfàs del Pi, Alicante: Universitat de València.

Fernández-Pampillón,A.M.; Domínguez, E., \& Armas, I. (2012). Diez criterios para mejorar la calidad de los materiales didácticos digitales. VII Jornadas de Campus Virtual UCM, Madrid (España).

Frasca, G (2003). Simulation versus Narrative: Introduction to Ludology. En M. Wolf. y B. Perron (Eds.). Game Theory Reader (pp. 221235). New York: Routledge.

Gee, P. (2004). Lo que nos enseñan los videojuegos sobre el aprendizajeyel alfabetismo. Málaga: Aljibe.

George, A.M.; Rohr, L.E., \& Byrne, J. (2016). Impact of Nintendo Wii games on physical literacyin children: Motor skills, physical fitness, activity behaviors, and knowledge. Sports, 4(3), 1-10. doi: 10.3390/ sports4010003

Gimeno, J. (1988). El currículum: una reflexión sobre la práctica. Madrid: Morata.

Griffiths, M. (2002). The educational benefits of videogames. Education and Health, 20(3), 47-51.

Gros, B. (2007). Digital games in education: The design of game-based learning environments. Journal of research on technology in education, 40(1), 23-38.

Gros, B. (2008). Videojuegos y aprendizaje. Barcelona: Graó

Gros, B. (2009). Certezas e interrogantes acerca del uso de los videojuegos para el aprendizaje. Comunicación, 7(1), 251-264.

Guarro, A. (2005). Los procesos de cambio educativo en una sociedad compleja. Diseño, desarrollo e innovación del currículum. Madrid: Pirámide.

Hargreaves, A. (1995). Development and Desire: A Postmodern Perspective. En T. R. Guskey y M. Huberman (Eds.).Professional Development in Education: New Paradigms and Practices (pp. 934). Nueva York: Tabacars College Press.

Jenny, S.E.; Schary, D.P.; Noble, K.M., \& Hamill, S.D. (2017). The Effectiveness of Developing Motor Skills Through Motion-Based Video Gaming: A Review. Simulation \& Gaming, 48(6), 722-734. doi.org/10.1177/1046878117738552

Juul, J. (2005). Games Telling Stories? En J. Raessens y J. Goldstein (Eds.). The handbook of computer games studies (pp. 219-226). Cambridge: Press Cambridge.

Kirk, D. (1990). School knowledge and the curriculum package-as-text. Journal of CurriculumStudies, 22, 409-425.

Lacasa, P. (2011). Losvideojuegos: Aprender en mundos reales y virtuales. Madrid: Morata.

Marqués, P. (2001). Selección de materiales didácticos y diseño de intervenciones educativas. Recuperado de:http:// peremarques.pangea.org/orienta.htm. Fecha de consulta: 15/01/2018.
Martínez Bonafé, J. (1991a). Proyectos curriculares y práctica docente. Sevilla: Díada.

Martínez Bonafé, J. (1991b). El cambio profesional mediante los materiales. Cuadernos de Pedagogía, 189, 61-64.

Molina, J.P. (2015). El uso de materiales curriculares impresos en la Educación Física de la ESO en la Comunidad Valenciana. Tesis Doctoral: Universitat de València.

Molina, J.P.; Devís, J., \&Peiró, C. (2008). Materiales curriculares: clasificación y uso en Educación Física. Pixel Bit. Revista de mediosy educación, 33, 183-197.

Molina, P.; Martínez-Baena, A., \& Gómez-Gonzalvo, F. (2017). El uso de materiales curriculares en Educación Física: racionalidad y desarrollo profesional del profesorado. VIREF, Revista de Educación Física, 6(3), 9-28.

Peiró, C., \&Devís, J. (1994). Materiales curriculares y recursos didácticos. En J. Díaz (Coord.).Temario de oposiciones al cuerpo de profesores de secundaria (pp. 11/1-11/18). Barcelona: Inde.

Pérez-López, I. J., \& Rivera García, E. (2017). Formar docentes, formar personas: análisis de los aprendizajes logrados por estudiantes universitarios desde una experiencia de gamificación. Signo y Pensamiento, 36(70), 112-129.

Pérez-López, I. J.; Rivera García, E., \& Trigueros Cervantes, C. (2017). «La profecía de los elegidos»: un ejemplo de gamificación aplicado a la docencia universitaria. Revista Internacional de Medicina y Ciencias de la Actividad Física y del Deporte, 17, 243-260.

Revuelta, I., \& Guerra, J. (2012). ¿Qué aprendo con videojuegos? Una perspectiva de meta-aprendizaje del videojugador. RED. Revista de educación a distancia, 33. Recuperado de: http://www.um.es/ead/ $\mathrm{red} / 33$

Rosas et al. (2003). Beyond Nintendo: design and assessment of educational video games for first and second grade students. Computers and education, 40, 71-94.

Salas, K., \& Lewis, I. (2013).Identifying types of archivements. The18th International Conference on Computer Games, 23-30

SánchezAgustí, M. (2005). Redefinir la historia que se enseña a la luz de las TIC: un análisis sobre nuevas maneras de aprender Roma. En M.I.Vera y D. Pérez (Eds.). Formación de la ciudadanía. Las TIC y los nuevos problemas [libro electrónico]. Alicante: XV Simposio internacional de didáctica de las ciencias sociales.

Schön, D. A. (1992). La formación de los profesores reflexivos. Barcelona: Paidós

Shafer, D. M.; Carbonara, C. P., \& Popova, L. (2011). Spatial presence and perceived reality as predictors of motion-based video game enjoyment. Presence, 20(6), 591-619.

Skinner, B.F.(1958). Teaching machines. Science, 128, 968-977.

Yuste, R. (2012). Evaluación psicopedagógica de videojuegos. Actas I congreso internacional videojuegos y educación. Alfàs del Pi: Universidad de Valencia.

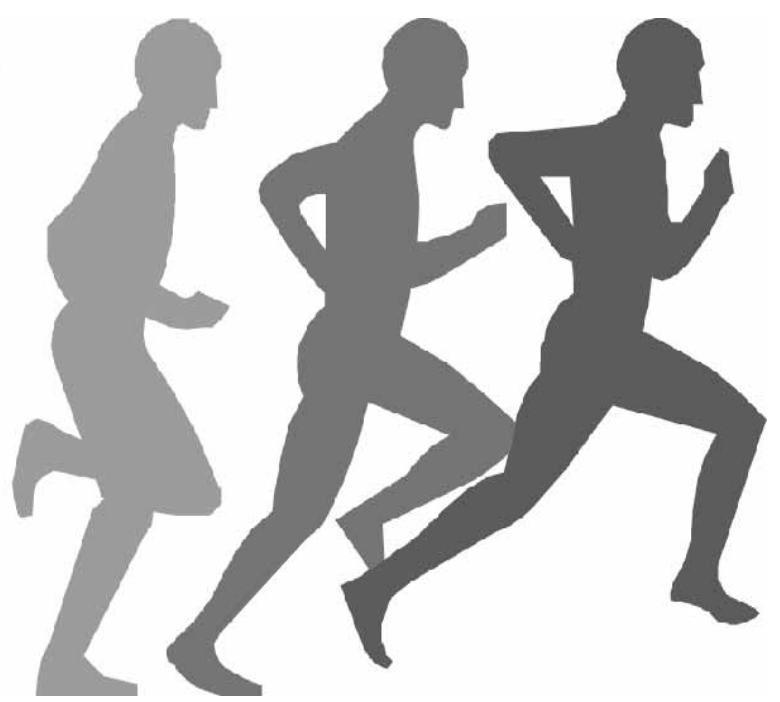

